\title{
Nipple-Areola Sparing Mastectomy Followed by Immediate Breast Reconstruction in 126 Patients
}

\author{
Xianming Wang, Min Wang, Jinkun Liu, Weicai Chen, Huisheng Wu, Shufeng Song
}

Center of Breast Diseases, Shenzhen Second People's Hospital, Shenzhen, China.

Email: wxm60@yahoo.com.cn

Received August $27^{\text {th }}, 2012$; revised September $26^{\text {th }}, 2012$; accepted October $4^{\text {th }}, 2012$

\begin{abstract}
This study is to explore the indications, procedures, effectiveness, and feasibility of nipple-areola sparing mastectomy followed by immediate breast reconstruction. The nipple-areola sparing mastectomy followed by immediate breast reconstruction was performed in 126 patients with breast cancer from June 2005 to October 2011. The cosmetic outcomes of the reconstructed breasts were evaluated according to objective and subjective criteria. Meanwhile, the postoperative complications were observed and the therapeutic efficacies were followed up. All the operations were successful. Six patients experienced mild complications early after surgery and were resolved after symptomatic treatment. Both the subjective and objective evaluation for the aesthetic outcomes yielded a satisfactory rate of $97.62 \%$ during the 6 80-month follow-up. No recurrence or metastasis was found in 118 cases. Nipple-areola sparing mastectomy followed by immediate breast reconstruction is a simple and effective option for significantly improving the cosmetic outcomes and quality of life of patients, without serious complications or impact on the comprehensive treatment and long-term effect against breast cancer.
\end{abstract}

Keywords: Breast Cancer; Nipple-Sparing Mastectomy; Breast Reconstruction

\section{Introduction}

In recent years, orthopedic techniques are being increasingly applied in the surgical treatment of breast cancer [1]. The St. Gallen 2011 Expert Consensus has recognized the cosmetic outcomes following extensive tumor resection aided by orthopedic techniques [2]. Not only does the reconstructive plastic surgery for breast cancer compensate the resected breast in mastectomy, but it also improve the safety and cosmetic outcomes of breastconserving surgery, thus improving the quality of life for patients. Evidence-based medicine has confirmed that skin-sparing mastectomy (SSM), comparably effective to traditional mastectomy in treating breast cancer, is associated with better cosmetic outcomes [3]. The nippleareola complex is essential in breast aesthetics. Nipple-sparing mastectomy (NSM) is subcutaneous mastectomy with nipple-areola complex left. A mastectomy that retains the nipple and areola does not only simplify breast reconstruction surgery, but significantly improves cosmetic outcomes. Although the technical improvement from SSM to NSM has been initiated, further research is required to determine the eligible population in view of the few long-term follow-up reports available. From June to 2005 to October 2010, 126 patients with breast cancer underwent nipple-areola sparing mastectomy followed by immediate breast reconstruction in our hospital, achieving satisfying outcomes. The cases are reported as follows.

\section{Materials and Methods}

\subsection{Study Design}

The trial was a prospective cohort study and was approved by the ethic committee review boards of Shenzhen Second People's Hospital, and enrollment began in June 2005. Patients signed the informed consent forms before operations.

\subsection{Participants}

The cohort included 126 female patients (Table 1) with breast cancer aged 28 to 56 years (mean 43 years). Based on TNM staging, there were 48 patients with stage 0 breast cancer, 23 cases with stage I stage 0 breast cancer, and 55 with stage II stage 0 breast cancer. Overall, there were 43 patients with ductal carcinoma in situ $(28$ with negative sentinel lymph node biopsy and 15 without axillary lymph node treatment), five patients with lobular carcinoma (two with negative sentinel lymph node biopsy and three without axillary lymph node treatment), 19 patients with stage I invasive ductal carcinoma, 52 
patients with stage II invasive ductal carcinoma, four patients with stage I invasive lobular carcinoma and three patients with stage II invasive lobular carcinoma. Ninety-four patients did not have axillary lymph node metastasis (including 42 with negative sentinel lymph node biopsy). Of the thirty-two who did, twenty-five had 1 to 3 lymph node metastases and seven had 4 or more. The molecular subtypes of the 78 cases of invasive carcinoma included 35 cases of LUMINAL A, 24 cases of LUMINAL B, 9 cases of HER-2 ENRICH and 10 cases of BASAL-LIKE.

\section{Inclusion Criteria}

1) Patients with stage 0 breast cancer with suspected multifocal lesions or extensive calcification range; residual tumor along the needle tract was suspected in 15 patients despite definite diagnosis via minimally invasive biopsy; non-Paget's disease; 2) Patients with stages I and II breast cancer (except T3N0 stage II breast cancer) with a margin between the tumor and the areola of $>2 \mathrm{~cm}$, and negative findings of subcutaneous tissues superior to the tumor and subareola tissues near the lesion through intraoperative rapid pathological examination; 3) Patients who had good overall conditions without serious heart disease or cerebrovascular disease, and could tolerate surgery; 4) Patients who met the requirements for breastconserving surgery but postoperative cosmetic expectations were poor due to small breast sizes; 5) Patients who chose mastectomy due to concerns about the risk of recurrence after breast-conserving surgery or unwillingness to receive postoperative radiation therapy; 6) Patients undergoing mastectomy in combination with prosthetic implantation, also known as breast reconstruction surgery.

\subsection{Surgical Methods}

All of the 126 patients underwent nipple-areola sparing breast cancer resection and primary reconstruction with silicone gel-filled breast implants. Incisions were made along the outer edge of the breast, the areola or folds under the breast following a curved shape for complete removal of the breast tissue from skin and the surface of pectoralis major. For patients whose incision was not located at the outer edge of the breast, an additional incision was made at the armpit for sentinel lymph node biopsy or axillary lymph node dissection. During separation beneath the nipple and areola, excessive division of tissues posterior to the nipple should be avoided to prevent postoperative ischemia and necrosis of the nipple and areola. The breast reconstruction should be terminated if positive pathological findings of the subcutaneous tissues under the tumor or subareola tissues near the lesion were present.
Table 1. Baseline characteristics of patients.

\begin{tabular}{cc}
\hline Characteristic & Patients (N = 126) \\
\hline Age & 43 \\
Average & $28-56$ \\
Range & \\
TNM Staging & 48 \\
Stage 0 & 23 \\
Stage I & 55 \\
Stage II & \\
Pathology & 43 \\
Ductal carcinoma in situ & 5 \\
Lobular carcinoma in situ & 19 \\
Stage I invasive ductal carcinoma & 52 \\
Stage II invasive ductal carcinoma & 4 \\
Stage I invasive lobular carcinoma & 3 \\
Stage II invasive lobular carcinoma & \\
Axillary Lymph Node & 94 \\
Positive & 32 \\
Negative &
\end{tabular}

Prosthesis was implanted in the posterior space of the pectoral muscle. The implant volume was calculated based on preoperative measurement of the contralateral breast. According to the measured volume of the contralateral breast, a selected water-filled balloon was placed into the space between the two chest muscles ipsilaterally during surgery. In the present cohort, $85 \%$ patients had relatively smaller breasts, and the volumes of used prostheses ranged from 180 to $240 \mathrm{~mL}$. The space between pectoralis major and pectoralis minor muscles, particularly the lower edges, was completely divided and part of the attachment of the pectoralis major to ribs was detached to prevent asymmetrical appearance of the breasts due to shift up of the prosthesis. Sufficient pads should be used to support the upper and lateral sides of the prosthesis during fixed dressing, and elastic tape was used to simultaneously fix both the upper and lower sides of both breasts in an oval shape so that both breasts were at the same level. Intense activity of the upper limbs was restricted in two weeks following the operation.

\subsection{Aesthetic Evaluation of Breast Reconstruction}

\subsubsection{Morphological Assessment of Reconstructed Breasts}

The appearance was classified according to the standards of a previous report [4] as: 1) Excellent: breasts were symmetrical, horizontal difference between nipples $\leq 2$ 
$\mathrm{cm}$; normal appearance without significant difference in shape compared with the contralateral breast; no breast lift or deformation due to scar tissues, feel was good and skin was normal; 2) Good: breasts were symmetrical, horizontal difference between nipples $\leq 3 \mathrm{~cm}$; normal appearance or slightly smaller shape than the contralateral breast; feel was slightly odd; and skin color became lighter and then glossy; 3) Poor: breasts were asymmetric; horizontal difference between nipples $>3 \mathrm{~cm}$; obviously deformed appearance and significantly smaller compared with the contralateral side; feel was bad; skin was thick and rough with rubber-like texture.

\subsubsection{Patient Self-Assessment}

Questionnaires were distributed among the patients 6 and 12 months after surgery, respectively, to determine their satisfaction with breast reconstruction.

\subsection{Postoperative Adjuvant Therapy}

According to the clinical staging and molecular biological subtypes specified in NCCN guidelines, postoperative adjuvant radiotherapy, chemotherapy, endocrine therapy and anti-HER-2 therapy were prescribed for all 126 patients. Conventional radiotherapy was administered for seven patients who had four or more lymph node metastases, and postoperative radiotherapy was given to six out of nine patients who had one to three lymph node metastases. The presence of prostheses did not affect the effectiveness of chemotherapy. Conformal radiotherapy of the clavicle region could be prescribed for patients with three or more lymph node metastases found in pathological examination without affecting the prostheses [5].

\section{Results}

\subsection{Postoperative Evaluation of Appearance}

The satisfaction survey from six to twelve months after surgery revealed that, of the 126 patients, 89 reported an "excellent" morphological assessment of breast reconstruction $(70.63 \%)$, while 34 reported a "good" result (26.98\%). Both subjective and objective evaluation measures were at a satisfactory level $(97.62 \%)$. Three patients reported a "bad" outcome $(2.38 \%)$, of which one experienced significant prosthetic displacement at the early stage of surgery and two developed prosthetic capsular contracture during postoperative radiotherapy.

\subsection{Postoperative Complications}

Early after surgery, a slightly dry, solid and black appearance of the nipples was observed in three patients due to poor blood supply. Although the wounds were healed spontaneously without special treatment, they were slightly smaller compared with the contralateral ones. Subcutaneous hematoma was observed at the skin folds under the nipples of two patients, and the wounds were closed after removal of hematoma and pressure bandage. One patient presented prosthesis displacement. None of the remaining patients experienced flap necrosis, effusion, wound dehiscence or other complications; primary closure of the wounds was achieved.

\subsection{Follow-Up}

The patients were followed up for 6 to 12 months and $97.62 \%$ of them were satisfied with the breast reconstruction outcomes following surgery. In the follow-up for 6 to 80 months, 15 patients $(11.9 \%)$ had recurrence or distant metastases, of which eight were regional recurrence $(6.34 \%$, including six cases of HER-2 ENRICH and two of BASAL-LIKE) and seven were distant metastases in 36 months after surgery $(5.55 \%$, including five cases of HER-2 ENRICH and two of BASAL-LIKE). Five of those patients died.

Another follow-up of 132 patients with stages $0, \mathrm{I}$ and II breast cancer who underwent breast-sparing surgery over the same period revealed 17 patients $(12.9 \%)$ of recurrence or distant metastases, of which nine were regional recurrence $(6.81 \%$, including seven cases of HER-2 ENRICH and two of BASAL-LIKE) six seven were distant metastases in 36 months after surgery (4.54\%, including four cases of HER-2 ENRICH and one of BASAL-LIKE). Four of those patients died.

\section{Discussion}

The development of breast cancer therapy in the $21 \mathrm{st}$ century has been steered by the important role of molecular spectra. The current consensus is also built upon multi-disciplinary treatment by various means aided by molecular classification of breast cancer. In recent years, breast-conserving surgery has gradually been applied across China, whereas the incidence of this technique witnesses a decline in Europe and the United States [6,7]. This could be mainly explained by the following aspects: 1) The increasing application of BRCA genetic testing has increased public awareness of prevention against this condition; and preventive mastectomy with reconstruction has been used in clinical settings; 2) The universal application of MRI and other imaging techniques has increased the detection rate of many previously occult lesions; 3) The addition of reconstructive plastic techniques in the surgical treatment of breast cancer does not only compensate the resected breast in mastectomy, but it also improves the safety and cosmetic outcomes of breast-conserving surgery, thus improving the quality of life for patients.

Evidence-based medicine has confirmed that skinsparing mastectomy is comparably effective to traditional 
mastectomy in treating breast cancer, and there is consensus that the extension of skin excision is independent of local recurrence, so the skin-sparing technique is not associated with an increased risk of local recurrence of breast cancer [8,9]. The study of Fersis et al. [3] has shown that postoperative local recurrence is mainly caused by residual breast ductal epithelium cells rather than breast skin tissues. Therefore, both approaches have a comparable local recurrence rate after operation. The nipple-areola complex (NAC) is an important part of the female breasts, and salvage of the NAC is vital for the cosmetic outcomes of breast reconstruction and postoperative quality of life. Laronga et al. [10] suggested a correlation between the involvement of the nipple-areola complex and lymph node metastasis, as well as the distance from the tumor to the edge of the areola, independent of tumor size, histological type and receptors. Therefore, nipple-areola sparing mastectomy is possible in some breast cancer patients for whom the resection of breast skin and the complex is unnecessary. In a clinical trial, Gerber et al. [11] have shown that the retention of the nipple-areola complex does not increase the risk of relapse if the margin of lesions is farther than $2 \mathrm{~cm}$ from the nipples. In a retrospective cohort study evaluating the onset age, tumor size, axillary lymph node status, hormone receptor status and HER-2 expression status of 47 patients undergoing the nipple-areola sparing modified radical surgery, Wang Shengying et al. [12] found no significant difference between the two groups in terms of overall survival (OS), disease-free survival (DFS) and local recurrence rate. This is consistent with the findings in our follow-up comparison between the present cohort and patients undergoing breast-conserving surgery over the same period.

Breast reconstruction following mastectomy can be divided into immediate (primary) and secondary operations. Compared with the secondary approach, immediate breast reconstruction is advantageous $[13,14]$ in: 1) achieving mastectomy and breast reconstruction simultaneously so that patients will not "feel a loss" after the initial operation; 2) reducing the required number of operations and overall cost and avoiding pain from a second surgery by accomplishing two procedures at one blow; and 3) having no interference with postoperative radiotherapy, chemotherapy and endocrine therapy. Based on the source of materials used for reconstruction, the surgery can be completed with either autogenous tissues or prosthetic implants. While autologous tissue reconstruction is usually associated with large wounds, difficult operations, high cost, obvious scars and high risk of postoperative complications, prosthesis is a safe, fast and simple option with little injury and short recovery period. Studies have confirmed that the implantation of prosthesis does not increase the risk of recurrence or develop- ment of breast cancer, and thus silicone gel prosthesis implantation is feasible and safe [11].

In the present study, we performed subcutaneous mastectomy sparing the nipple, areola and skin of breasts in strict compliance with the indication, and did not conduct primary prosthetic implantation until it was confirmed that no residual tumor cells were present beneath the nipple-areola complex. The subjective and objective evaluation of postoperative cosmetic outcomes was good in $97.62 \%$ of patients, which demonstrated the unique advantages of the technique in improving both aesthetic effects and the quality of life of patients. Moreover, the procedure did not affect the performance of postoperative adjuvant therapy. The key to breast reconstruction is the maintenance of symmetrical alignment of the skin folds posterior to both breasts. When the folds are stripped during mastectomy, the skin and the underlying tissues should be sutured and fixed to form an alternative structure. The breast folds should be such fixed that the distance from the areola to the folds is equal to the contralateral counterpart, otherwise the nipple may be prone to skew and the lower half of the breast may lack fullness [15]. Postoperative complications are common in patients undergoing traditional mastectomy. Three patients had compromised blood flow to the nipples, leading to partial atrophy, which could be due to improper retention of the vascular network in the subareolar layer. Crowe et al. [16] noted that improper operation was a major cause of necrosis of the retained nipple-areola complex. The blood supply of the nipple-areola complex is mainly derived from two circulatory networks - deep blood vessels in the mammary gland and capillary network at the subareolar layer. During operation of nipple-areola sparing mastectomy, the deeper supply is interrupted and all blood supply comes from the capillary network at the layer 3 - $5 \mathrm{~mm}$ from the areola epidermis, which should be protected [17]. Prosthesis displacement, a major risk factor of poor cosmetic outcomes, was present in one patient in the present cohort, as a result of excessive division of the posterior space to the pectoralis major and improper postoperative fixation. Such displacement can be effectively prevented by cutting off a part of the rib attachment points of the pectoralis major to place the lower edge of the prosthesis in the pectoralis major fissures, so that the lower half of breasts can appear to be nice and full. Postoperative radiotherapy has a certain negative impact on breast reconstruction (two patients experienced capsular contracture of the prosthesis). Hence, tissue spacers may be implanted for patients with a clear indication for radiotherapy, and replaced by the prosthesis through secondary operation after the completion of radiotherapy.

\section{Conclusion}

Based on the experience of the 126 patients undergoing 
nipple-areola sparing mastectomy (NSM) with primary prosthesis implantation, we have found this technique to be a simple and effective option for significantly improving the cosmetic outcomes and quality of life of patients, without serious complications or impact on the comprehensive treatment and long-term effect against breast cancer. It is an effective option for surgical treatment of breast cancer under clinical settings.

\section{Acknowledgements}

The study was supported by funds from Shenzhen $\mathrm{Mu}-$ nicipal Bureau of Science and Technology (200801004).

\section{REFERENCES}

[1] F. Fitzal and P. Schrenk, "Oncoplastic Breast Surgery: A Guide to Clinic Practice," Springer Wien, New York, 2010. doi:10.1007/978-3-211-99317-0

[2] A. Goldhirsch, J. N. Ingle and R. D. Gelber, "Thresholds for Therapies: Highlights of the St Gallen International Expert Consensus on the Primary Therapy of Early Breast Cancer 2009," Annals of Oncology, Vol. 20, No. 8, 2009, pp. 1319-1329.

[3] N. Fersis, A. Hoenig, K. Relakis, et al., "Skin Sparing Mastectomy and Immediate Breast Reconstruction: Incidence of Recurrence in Patients with Invasive Breast Cancer," Breast, Vol. 13, No. 6, 2004, pp. 488-493. doi:10.1016/j.breast.2004.06.009

[4] B. N. Zhang, T. Zhang, W. G. Hu, et al., "The BreastPreserving Surgery for Breast Cancer," Zhong Liu Xue Za Zhi, Vol. 8, No. 6, 2002, pp. 311-314.

[5] H. C. Jiang, F. C. Li and J. Li, "Skin-Sparing Incision Mastectomy and Immediate Implanting Breast Reconstruction," Zhong Guo Shi Yong Wai Ke Za Zhi, Vol. 25, No. 2, 2005, pp. 84-85.

[6] M. Morrow, "More Mastectomies: Is This What Patients Really Want?" Journal of Clinical Oncology, Vol. 27, No. 25, 2009, pp. 4038-4040. doi:10.1200/JCO.2009.23.0078

[7] Balch, Jacobs: "Mastectomies on the Rise for Breast Cancer: The Tide Is Changing," Annals of Surgical Oncology, Vol. 16, No. 10, 2009, pp. 2669-2672 doi:10.1245/s10434-009-0634-y

[8] H. Y. Wang, R. S. Ali, S. C. Chen, et al., "One-Stage Immediate Breast Reconstruction with Implant Following Skin-Sparing Mastectomy in Asian Patients," Annals of
Plastic Surgery, Vol. 60, No. 4, 2008, pp. 362-366. doi:10.1097/SAP.0b013e318063ef70

[9] H. Medina-Franco, L. O. Vasconez, R. J. Fix, et al., "Factors Associated with Local Recurrence after Skin-Sparing Mastectomy and Immediate Breast Reconstruction for Invasive Breast Cancer," Annals of Surgery, Vol. 235, No. 6, 2002, pp. 814-819. doi:10.1097/00000658-200206000-00008

[10] C. Laronga, B. Kemp, D. Johnston, et al., "The Incidence of Occult Nipple-Areola Complex Involvement in Breast Cancer Patients Receiving a Skin-Sparing Mastectomy," Annals of Surgical Oncology, Vol. 6, No. 6, 1999, pp. 609-613. doi:10.1007/s10434-999-0609-Z

[11] B. Gerber, A. Krause, T. Reimer, et al., "Skin-Sparing Mastectomy with Conservation of the Nipple-Areola Complex and Autologous Reconstruction Is an Oncologically Safe Procedure," Annals of Surgery, Vol. 238, No. 1, 2003, pp. 120-127. doi:10.1097/00000658-200307000-00016

[12] S. Y. Wang, Z. Z. Zhu, D. F. Peng, et al., "Efficacy of Modified Radical Operation of Preserving Nipple-Areolar Complex on Early Stage Breast Cancer," Ai Zheng, Vol. 28, No. 3, 2009, pp. 41-45.

[13] N. Fersis, A. Hoenig, K. Relakis, et al., "Skin-Paring Mastectomy and Immediate Breast Reconstruction: Incidence of Reurrence in Patients with Invasive Breast Cancer," Breast, Vol. 13, No. 6, 2004, pp. 488-493. doi:10.1016/j.breast.2004.06.009

[14] K. J. Downes, B. S. Glatt, S. K. Kanchwala, et al., "Skin-Paring Mastectomy and Immediate Breast Reconstruction Is an Acceptable Treatment Option for Patients with High-Risk Breast Carcinoma," Cancer, Vol. 103, No. 5, 2005, pp. 906-913. doi:10.1002/cncr.20851

[15] J. H. Fu, B. Ke, C. X. Yang, et al., "Modified Radical Mastectomy by Reserving Skin and Nipple Combined with Breast Reconstruction: A Report of 32 Cases," Zhong Guo Shi Yong Wai Ke Za Zhi, Vol. 25, No. 1, 2005, pp. 54-55.

[16] J. P. Crowe, J. A. Kim, R. Yetman, et al., "Nipple-Sparing Mastectomy: Technique and Results of 54 Procedures," Archives of Surgery, Vol. 139, No. 2, 2004, pp. 148-150. doi:10.1001/archsurg.139.2.148

[17] D. Z. Gao, J. Z. Sun, Q. S. Yin, et al., "A Study of the Blood Supply Relevant to Prevention of Nipple-Areola Necrosis in Female Breast Operation," Zhong Guo Pu Tong Wai Ke Za Zhi, Vol. 14, No. 4, 2005, pp. 269-272 